\title{
Atomistic Simulation of Protein Encapsulation in Metal-Organic Frameworks
}

\author{
Haiyang Zhang, ${ }^{\dagger *}$ Yongqin Lv, ${ }^{\dagger *}$ Tianwei Tan, ${ }^{\dagger *}$ and David van der Spoel ${ }^{\S}$
}

${ }^{\dagger}$ Beijing Key Laboratory of Bioprocess, College of Life Science and Technology, Beijing University of Chemical Technology, Box 53, 100029 Beijing, China

${ }^{\ddagger}$ Department of Biological Science and Engineering, School of Chemistry and

Biological Engineering, University of Science and Technology Beijing, 100083

Beijing, China

${ }^{\S}$ Uppsala Center for Computational Chemistry, Science for Life Laboratory, Department of Cell and Molecular Biology, Uppsala University, Husargatan 3, Box 596, SE-75124 Uppsala, Sweden

Corresponding author: 1vyq@mail.buct.edu.cn (Y.L.); twtan@mail.buct.edu.cn (T.T) 


\section{Contents}

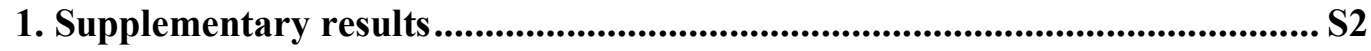

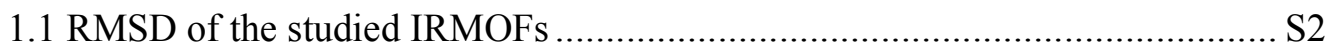

$1.2 R_{\mathrm{g}}$ of AA side chains and hydrophobicity scale of AAs .............................. S3

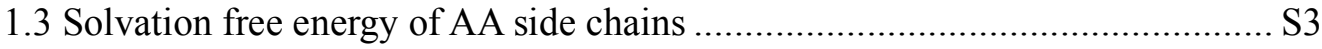

$1.4 \Delta G$ of AA side chains with IRMOF-74-I..................................................... S5

1.5 Water distribution in the pore channel of the IRMOFs ................................ S6

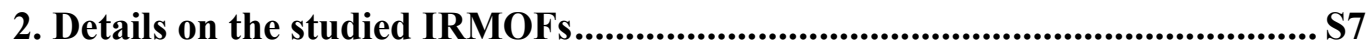

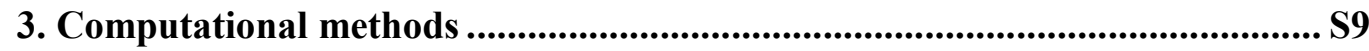

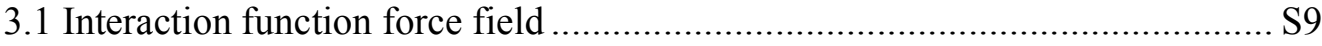

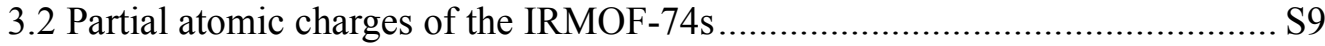

3.3 Evaluation of $\Delta G$ between AA side chains and IRMOF-74-I ..................... S12

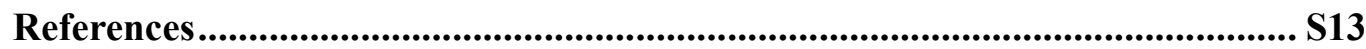




\section{Supplementary results}

\subsection{RMSD of the studied IRMOFs}

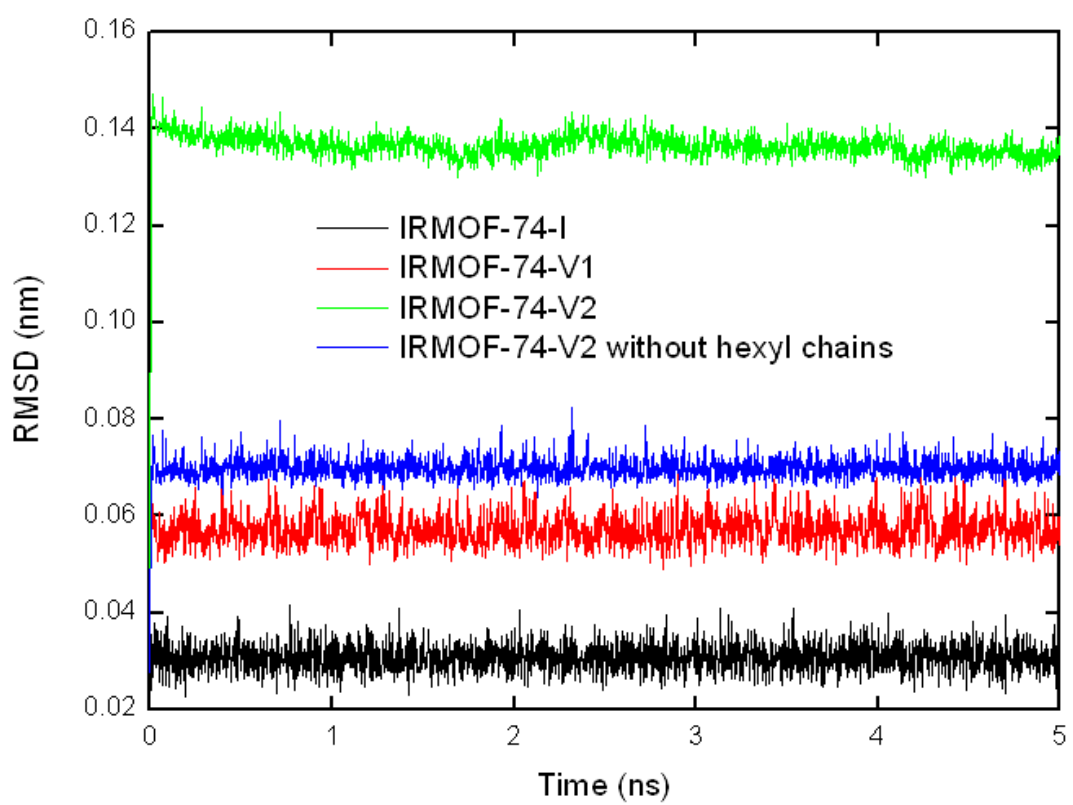

Figure S1. Root-mean-square deviation (RMSD) of the studied IRMOFs from their own crystal structure during 5-ns simulations.

Figure S1 shows RMSDs of the studied IRMOFs from their own crystal structure during 5-ns simulations. The MOF structures were stable and well maintained during the simulations, providing high credit for the force field used. Larger RMSD values ( $0.14 \mathrm{~nm}$ ) of IRMOF-74-V2 are resulted from flexible hexyl chains and the removal of these chains gives a stable and smaller fluctuation $(\sim 0.07 \mathrm{~nm})$. 


\section{$1.2 R_{\mathrm{g}}$ of AA side chains and hydrophobicity scale of AAs}

Table S1. Radius of gyration $\left(R_{\mathrm{g}}\right)$ of AA side chains and hydrophobicity scale of AAs

\begin{tabular}{|c|c|c|c|c|c|c|c|}
\hline \multirow{2}{*}{$\begin{array}{l}\text { Side chain } \\
\text { analog }\end{array}$} & \multicolumn{2}{|l|}{$R_{\mathrm{g}}$} & \multirow{2}{*}{ Amino acid } & \multirow{2}{*}{ Abbr. } & \multicolumn{3}{|c|}{ Hydrophobicity } \\
\hline & $\mathrm{AA}$ & quadAA & & & kd scale $^{a}$ & ww scale ${ }^{b}$ & hh scale \\
\hline Ala & 0.05 & 0.15 & Ala & A & 1.8 & -0.17 & 0.11 \\
\hline Ser & 0.08 & 0.20 & Ser & $\mathrm{S}$ & -0.8 & -0.13 & 0.84 \\
\hline Thr & 0.12 & 0.23 & Thr & $\mathrm{T}$ & -0.7 & -0.14 & 0.52 \\
\hline Val & 0.09 & 0.24 & Val & $\mathrm{V}$ & 4.2 & -0.07 & -0.31 \\
\hline Cys & 0.13 & 0.24 & Cys & $\mathrm{C}$ & 2.5 & 0.24 & -0.13 \\
\hline Asn & 0.13 & 0.28 & Asn & $\mathrm{N}$ & -3.5 & -0.42 & 2.05 \\
\hline Ile & 0.16 & 0.29 & Ile & I & 4.5 & 0.31 & -0.60 \\
\hline Leu & 0.14 & 0.31 & Leu & $\mathrm{L}$ & 3.8 & 0.56 & -0.55 \\
\hline Hie & 0.16 & 0.35 & Hic & $\mathrm{H}$ & 32 & 096 & \\
\hline Hid & 0.16 & 0.35 & HIS & $\mathrm{H}$ & -3.2 & -0.90 & 2.00 \\
\hline Met & 0.15 & 0.37 & Met & $\mathrm{M}$ & 1.9 & 0.23 & -0.10 \\
\hline Gln & 0.16 & 0.37 & Gln & $\mathrm{Q}$ & -3.5 & -0.58 & 2.36 \\
\hline Phe & 0.18 & 0.39 & Phe & $\mathrm{F}$ & 2.8 & 1.13 & -0.32 \\
\hline Tyr & 0.20 & 0.42 & Tyr & $\mathrm{Y}$ & -1.3 & 0.94 & 0.68 \\
\hline \multirow[t]{7}{*}{$\operatorname{Trp}$} & 0.21 & 0.44 & $\operatorname{Trp}$ & $\mathrm{W}$ & -0.9 & 1.85 & 0.30 \\
\hline & & & Gly & $\mathrm{G}$ & -0.4 & -0.01 & 0.74 \\
\hline & & & Pro & $\mathrm{P}$ & -1.6 & -0.45 & 2.23 \\
\hline & & & Asp & $\mathrm{D}$ & -3.5 & -1.23 & 3.49 \\
\hline & & & Glu & $\mathrm{E}$ & -3.5 & -2.02 & 2.68 \\
\hline & & & Lys & K & -3.9 & -0.99 & 2.71 \\
\hline & & & Arg & $\mathrm{R}$ & -4.5 & -0.81 & 2.58 \\
\hline DHEH & & 0.34 & & & & & \\
\hline DREK & & 0.40 & & & & & \\
\hline${ }^{a}$ Taken fror & f. ${ }^{1}$; & ref. $^{2} ;{ }^{c} \mathrm{fr}$ & & & & & \\
\hline
\end{tabular}

\subsection{Solvation free energy of AA side chains}

Solvation free energy of AA side chains are given in Table S2. The results are comparable with Shirts's work $^{4}$ and show similar derivations from experimental observations, validating the correctness of the protocol setup in the thermodynamic integration calculations. Since the difference between AMBER(ff94) and AMBER(ffSB) force fields is that the latter only replaced the backbone dihedrals 
$(\varphi / \psi)^{5}$ and this change is not relevant here, the agreement with Shirts's work is expected.

Table S2. Solvation free energy of AA side chains and comparison with experimental measurements and with Shirts's work

\begin{tabular}{|c|c|c|c|c|c|}
\hline \multirow{2}{*}{$\begin{array}{l}\text { Side chain } \\
\text { analog }\end{array}$} & \multirow{2}{*}{ Expt. } & \multicolumn{2}{|c|}{ Shirts's work ${ }^{4}$} & \multicolumn{2}{|l|}{ This work } \\
\hline & & AMBER(ff94) & Error & AMBER(ff99SB) & Error \\
\hline Ala & 1.94 & $2.57 \pm 0.02$ & 0.63 & $2.48 \pm 0.03$ & 0.54 \\
\hline Val & 1.99 & $2.69 \pm 0.03$ & 0.70 & $2.43 \pm 0.04$ & 0.44 \\
\hline Leu & 2.28 & $2.72 \pm 0.03$ & 0.44 & $2.38 \pm 0.10$ & 0.10 \\
\hline Ile & 2.15 & $2.84 \pm 0.03$ & 0.69 & $2.50 \pm 0.06$ & 0.35 \\
\hline Ser & -5.06 & $-4.37 \pm 0.02$ & 0.69 & $-4.43 \pm 0.03$ & 0.63 \\
\hline Thr & -4.88 & $-3.83 \pm 0.03$ & 1.05 & $-4.06=$ & 0.82 \\
\hline Phe & -0.76 & $0.10 \pm 0.04$ & 0.86 & $-0.26 \pm 0.06$ & 0.50 \\
\hline Tyr & -6.11 & $-4.23 \pm 0.04$ & 1.88 & $-4.56 \pm 0.16$ & 1.55 \\
\hline Cys & -1.24 & $0.11 \pm 0.02$ & 1.35 & $-0.10 \pm 0.08$ & 1.14 \\
\hline Met & -1.48 & $0.91 \pm 0.03$ & 2.39 & $0.56 \pm 0.07$ & 2.04 \\
\hline Asn & -9.68 & $-7.80 \pm 0.03$ & 1.88 & $-9.60 \pm 0.07$ & 0.08 \\
\hline Gln & -9.38 & $-7.69 \pm 0.04$ & 1.69 & $-10.10 \pm 0.05$ & -0.72 \\
\hline Trp & -5.88 & $-4.88 \pm 0.05$ & 1.00 & $-5.14 \pm 0.09$ & 0.74 \\
\hline Hid & -10.27 & $-8.43 \pm 0.04$ & 1.84 & $-8.49 \pm 0.05$ & 1.78 \\
\hline \multirow[t]{2}{*}{ Hie } & -10.27 & $-8.98 \pm 0.04$ & 1.29 & $-9.15 \pm 0.10$ & 1.12 \\
\hline & & \multicolumn{2}{|c|}{ Average error: $1.23 \pm 0.57$} & \multicolumn{2}{|c|}{ Average error: $0.74 \pm 0.69$} \\
\hline
\end{tabular}




\section{$1.4 \Delta G$ of AA side chains with IRMOF-74-I}

$\Delta G$ for AA and quad-AA side chains are given in Tables S3 and S4, respectively. The thermodynamic cycle used for calculation of $\Delta G$ refers to the section of Computational methods below.

Table S3. Binding free energy $(\mathrm{kcal} / \mathrm{mol})$ of AA side chains with IRMOF-74-I ${ }^{a}$

\begin{tabular}{|c|c|c|c|c|c|c|}
\hline \multirow{2}{*}{$\begin{array}{l}\text { Side } \\
\text { chain } \\
\text { analog }\end{array}$} & \multicolumn{2}{|c|}{$\Delta G_{\text {complexation }}^{\mathrm{MOF}-\mathrm{AA}}$} & \multicolumn{2}{|c|}{$\Delta G_{\mathrm{sol}}^{\mathrm{AA}}$} & \multicolumn{2}{|c|}{$\Delta G_{\text {complexation }}^{\mathrm{MOF}-\mathrm{AA}}-\Delta G_{\mathrm{sol}}^{\mathrm{AA}}$} \\
\hline & $\Delta G_{\text {coul }}$ & $\Delta G_{\mathrm{vdw}}$ & $\Delta G_{\text {coul }}$ & $\Delta G_{\mathrm{vdw}}$ & $\Delta G_{\text {coul }}$ & $\Delta G_{\mathrm{vdw}}$ \\
\hline ALA & $-0.02 \pm 0.00$ & $3.16 \pm 0.06$ & $0.00 \pm 0.00$ & $2.49 \pm 0.03$ & $-0.02 \pm 0.00$ & $0.67+0.07$ \\
\hline SER & $-5.78 \pm 0.05$ & $0.74 \pm 0.05$ & $-6.1 \pm 0.01$ & $1.67 \pm 0.03$ & $0.32 \pm 0.05$ & $-0.93 \pm 0.06$ \\
\hline THR & $-5.92 \pm 0.05$ & $1.92 \pm 0.13$ & $-5.81 \pm 0.01$ & $1.75 \pm 0.06$ & $-0.11 \pm 0.05$ & $0.17 \pm 0.14$ \\
\hline VAL & $-2.04 \pm 0.01$ & $1.62 \pm 0.09$ & $-2.02 \pm 0.00$ & $1.92 \pm 0.08$ & $-0.02 \pm 0.01$ & $-0.30 \pm 0.12$ \\
\hline CYS & $-0.01 \pm 0.00$ & $1.84 \pm 0.08$ & $-0.02 \pm 0.00$ & $2.45 \pm 0.04$ & $0.01 \pm 0.00$ & $-0.61 \pm 0.09$ \\
\hline ASN & $-10.57 \pm 0.05$ & $-0.49 \pm 0.11$ & $-10.82 \pm 0.01$ & $1.22 \pm 0.07$ & $0.25 \pm 0.05$ & $-1.71 \pm 0.13$ \\
\hline ILE & $0.01 \pm 0.00$ & $3.74 \pm 0.15$ & $0.00 \pm 0.00$ & $2.50 \pm 0.06$ & $0.01 \pm 0.00$ & $1.24 \pm 0.16$ \\
\hline LEU & $-0.05 \pm 0.00$ & $1.19 \pm 0.17$ & $-0.05 \pm 0.00$ & $2.43 \pm 0.10$ & $0.00 \pm 0.00$ & $-1.24 \pm 0.20$ \\
\hline HID & $-9.38 \pm 0.05$ & $-1.74 \pm 0.14$ & $-9.84 \pm 0.02$ & $0.69 \pm 0.10$ & $0.46 \pm 0.05$ & $-2.43 \pm 0.17$ \\
\hline HIE & $-8.85 \pm 0.06$ & $-1.83 \pm 0.14$ & $-9.31 \pm 0.01$ & $0.82 \pm 0.05$ & $0.46 \pm 0.06$ & $-2.65 \pm 0.15$ \\
\hline MET & $-1.34 \pm 0.03$ & $-0.85 \pm 0.10$ & $-1.58 \pm 0.00$ & $2.14 \pm 0.07$ & $0.24 \pm 0.03$ & $-2.99 \pm 0.12$ \\
\hline GLN & $-14.56 \pm 0.25$ & $-0.44 \pm 0.10$ & $-11.41 \pm 0.02$ & $1.31 \pm 0.05$ & $-3.15 \pm 0.25$ & $-1.75 \pm 0.11$ \\
\hline PHE & $-1.54 \pm 0.03$ & $-3.34 \pm 0.11$ & $-2.31 \pm 0.01$ & $2.06 \pm 0.06$ & $0.77 \pm 0.03$ & $-5.40 \pm 0.13$ \\
\hline TYR & $-5.32 \pm 0.12$ & $-2.27 \pm 0.20$ & $-6.12 \pm 0.01$ & $1.56 \pm 0.16$ & $0.80 \pm 0.12$ & $-3.83 \pm 0.26$ \\
\hline TRP & $-5.56 \pm 0.09$ & $-3.92 \pm 0.20$ & $-6.21 \pm 0.01$ & $1.07 \pm 0.08$ & $0.65 \pm 0.09$ & $-4.99 \pm 0.22$ \\
\hline
\end{tabular}

${ }^{a}$ standard deviations equal to 0.00 indicate a value smaller than 0.01 
Table S4. Binding free energy $(\mathrm{kcal} / \mathrm{mol})$ of quad-AA side chains with IRMOF-74-I ${ }^{a}$

\begin{tabular}{|c|c|c|c|c|c|c|}
\hline \multirow{2}{*}{$\begin{array}{l}\text { Quad } \\
\text { side } \\
\text { chain } \\
\text { analog }\end{array}$} & \multicolumn{2}{|c|}{$\Delta G_{\text {complexation }}^{\text {MOF-quadAA }}$} & \multicolumn{2}{|c|}{$\Delta G_{\mathrm{sol}}^{\text {quadAA }}$} & \multicolumn{2}{|c|}{$\Delta G_{\text {complexation }}^{\text {MOF-quadAA }}-\Delta G_{\text {sol }}^{\text {quadAA }}$} \\
\hline & $\Delta G_{\text {coul }}$ & $\Delta G_{\mathrm{vdw}}$ & $\Delta G_{\text {coul }}$ & $\Delta G_{\mathrm{vdw}}$ & $\Delta G_{\text {coul }}$ & $\Delta G_{\mathrm{vdw}}$ \\
\hline ALA & $0.05 \pm 0.00$ & $2.19 \pm 0.14$ & \pm 0.00 & $=0.11$ & $0.04 \pm 0.00$ & .18 \\
\hline SER & $-20.9 \pm 0.44$ & $-4.95 \pm 0.17$ & $16.01 \pm 0.04$ & $-0.23 \pm 0.08$ & $-4.89 \pm 0.44$ & 0.19 \\
\hline THR & $-11.58 \pm 0.12$ & $-9.41 \pm 0.28$ & $-13.47 \pm 0.03$ & & & \\
\hline VAL & & & $-0.08 \pm 0.00$ & & & \\
\hline CYS & $-5.90 \pm 0.03$ & $-4.10 \pm 0.21$ & $-5.38 \pm 0.01$ & $1.30 \pm 0.09$ & $-0.52 \pm 0.03$ & $-5.40 \pm 0.23$ \\
\hline ASN & $-25.45 \pm 0.41$ & $-10.95 \pm 0.30$ & $-26.26 \pm 0.34$ & $-1.87=$ & & \\
\hline ILE & & & & & & \\
\hline LEU & $-0.66 \pm 0.02$ & $-11.68 \pm$ & $-0.19 \pm 0.00$ & 33 & -0.4 & -15 . \\
\hline HID & $-35.96 \pm 0.20$ & $-15.67 \pm 074$ & $-31.16 \pm 0.13$ & $-1.60 \pm 0.13$ & $-4.80 \pm 0.24$ & $=0.75$ \\
\hline HIE & $-38.45 \pm 0.25$ & $-13.87 \pm 0.97$ & $-33.91 \pm 0.26$ & & & \\
\hline MET & $-7.07 \pm 0.07$ & $-11.08 \pm 0.78$ & $-5.67 \pm 0.01$ & & -1.4 & \pm 0.79 \\
\hline GLN & $-41.8 \pm 0.63$ & $-18.47 \pm 0.62$ & $-37.64 \pm 0.24$ & $-1.04 \pm 0.23$ & -4.16 & $-17.43 \pm 0.66$ \\
\hline PHE & & $-14.40 \pm 0.95$ & $-7.65 \pm 0.02$ & & $-0.05 \pm 0.37$ & $-16.99 \pm 0.97$ \\
\hline TYR & $-28.31 \pm 0.56$ & $-17.59+0.90$ & $-23.23 \pm 0.03$ & $0.78 \pm 0.16$ & $-5.08 \pm 0.56$ & $-18.37 \pm 0.91$ \\
\hline TRP & $-21.34 \pm 0.34$ & $-23.57 \pm 0.95$ & $-22.14 \pm 0.04$ & $-0.21 \pm 0.20$ & $0.80 \pm 0.34$ & $-23.36 \pm 0.97$ \\
\hline DHEH & $-91.01 \pm 0.16$ & $-18.03 \pm 1.09$ & $-87.87 \pm 0.16$ & $-2.48 \pm 0.11$ & $-3.14 \pm 0.23$ & $-15.55 \pm 1.10$ \\
\hline DREK & $-94.99 \pm 0.96$ & $-19.34 \pm 0.81$ & $-100.53 \pm 2.2$ & $-1.98 \pm 0.12$ & $5.54 \pm 2.40$ & $-17.36 \pm 0.82$ \\
\hline
\end{tabular}

${ }^{a}$ Standard deviations equal to 0.00 indicate a value smaller than 0.01

\subsection{Water distribution in the pore channel of the IRMOFs}

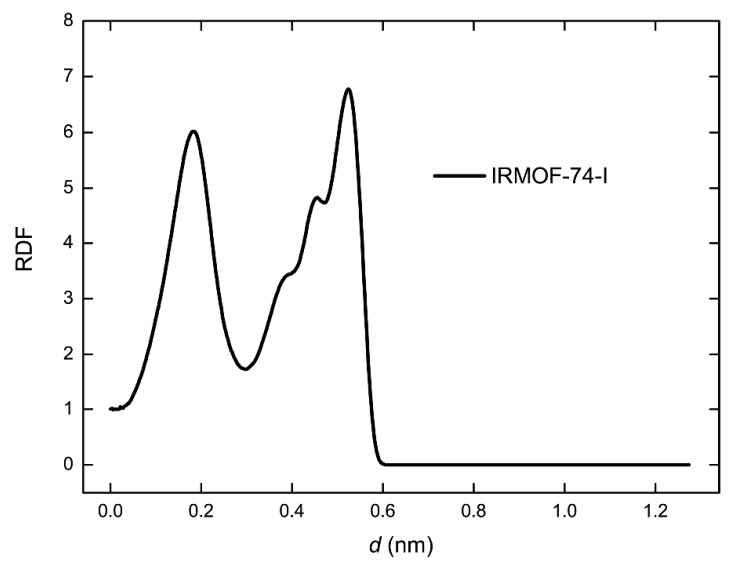

Figure S2. Radial distribution function (RDF) of water molecules around the MOF axis (i.e. $Z$-axis) in one channel of IRMOF-74-I. $d$ is the distance from the axis computed using $x$ - and $y$-components only, similar to the definition for radius $(R)$ of pore aperture in Figure 2a in the main text.

Radial distribution functions (RDF) of water molecules around the axis of the MOF 
pore channel reveal a higher density of water distribution near the wall of IRMOFs, as shown by the peaks of $\sim 0.5 \mathrm{~nm}$ in IRMOF-74-I (Figure S2).

\section{Details on the studied IRMOFs}

Table S5. Crystal information of the MOFs studied

\begin{tabular}{lccc}
\hline Name & Crystal cell size $\left(\mathrm{nm}^{3}\right)$ & Crystal cell angle $\left({ }^{\circ}\right)$ & CCDC code \\
\hline IRMOF-74-I & $2.60 \times 2.60 \times 0.68$ & & 668974 \\
IRMOF-74-V1 ${ }^{a}$ & $6.64 \times 6.64 \times 0.68$ & $(\alpha, \beta, \gamma)$ & 841645 \\
IRMOF-74-V1-HA & - & $=(90,90,120)$ & - \\
IRMOF-74-V1-DGA & - & - \\
IRMOF-74-V2 & & 841646 \\
${ }^{a}$ Termed as IRMOF-74-V in ref. & $6.90 \times 6.90 \times 0.64$ & \\
\hline
\end{tabular}

(a)

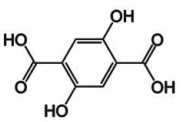

IRMOF-74-I
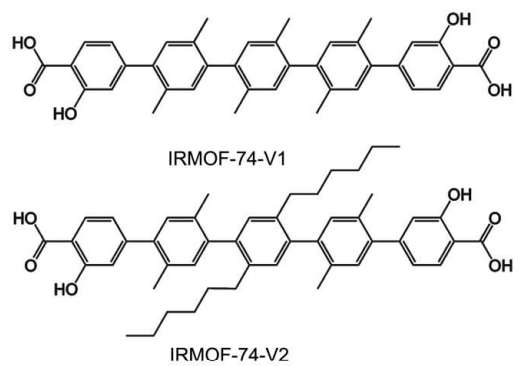

(b)

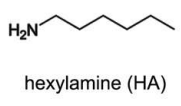

$\mathrm{H}_{2} \mathrm{~N} \sim \mathrm{O}$

diglycolamine (DGA)

(c)

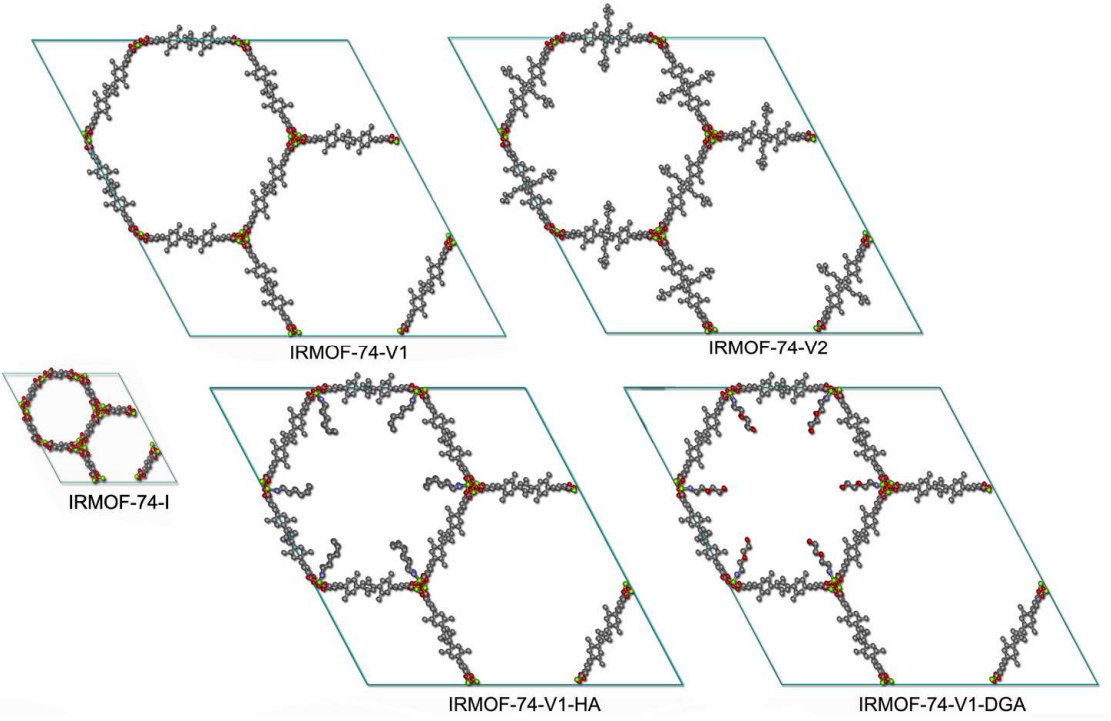

Figure S3. (a) Organic linkers, (b) modification groups, and (c) initial configurations of the five IRMOFs studied in this work. Hydrogen atoms are omitted for clarity. 
Crystal information of the five MOFs studied in this work is given in Table S5. IRMOF-74s display a triclinic crystal structure. IRMOF-74-I has a small pore channel suitable for amino acid (AA) inclusion, while IRMOF-74-V with a larger pore aperture enables encapsulation of peptide and mini-protein such as Trp-cage. Organic linkers of IRMOF-74-V1 can be functionalized by hexyl chains, ${ }^{6}$ giving a more hydrophobic channel. This modification produces IRMOF-74-V2 and slightly influences the crystal cell size of original IRMOF-74-V1 (Table S5).

Here we post-modified IRMOF-74-V1 using hexylamine (HA) or diglycolamine (DGA) chains by metal-chelating interactions of magnesium $(\mathrm{Mg})$ ions with amine groups. Molecular structure for organic linkers of the IRMOFs and for modification groups of HA and DGA are presented in Figures S3a and S3b, respectively. HA gives a hydrophobic modification and DGA a hydrophilic one. These crystallographic and post-modified IRMOF structures are displayed in Figure S3c. A schematic diagram for modification of IRMOF-74-V1 with DGA is shown in Figure S4.
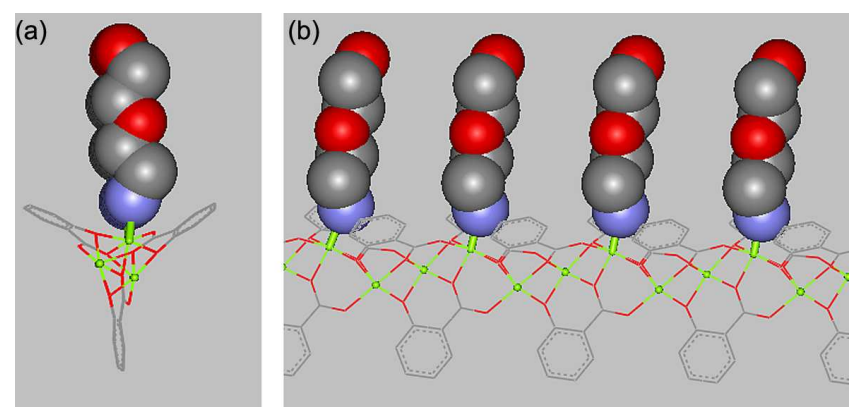

Figure S4. Top (a) and side (b) view of IRMOF-74-V1 modification with DGA. Mg atoms are shown with ball models in green and DGA with space-filling models. 


\section{Computational methods}

\subsection{Interaction function force field}

The nonbonded interaction between the atoms was calculated by the Coulomb and Lennard-Jones (LJ) potentials:

$$
V_{i j}=\frac{1}{4 \pi \varepsilon_{0}} \frac{q_{i} q_{j}}{r_{i j}}+4 \varepsilon_{i j}\left[\left(\frac{\sigma_{i j}}{r_{i j}}\right)^{12}-\left(\frac{\sigma_{i j}}{r_{i j}}\right)^{6}\right]
$$

Derivation of atomic charges for the MOFs studied was presented in the next section.

The Lennard-Jones parameters ( $\sigma$ and $\varepsilon$ ) for the MOF atoms are given in Table S6.

Table S6. Lennard-Jones parameters in the UFF for the MOF atoms

\begin{tabular}{lcc}
\hline Element & $\sigma(\mathrm{nm})$ & $\varepsilon(\mathrm{kJ} / \mathrm{mol})$ \\
\hline $\mathrm{C}$ & 0.3431 & 0.4396 \\
$\mathrm{H}$ & 0.2571 & 0.1842 \\
$\mathrm{O}$ & 0.3118 & 0.2512 \\
$\mathrm{Mg}$ & 0.2691 & 0.4647 \\
$\mathrm{~N}$ & 0.3261 & 0.2889 \\
\hline
\end{tabular}

\subsection{Partial atomic charges of the IRMOF-74s}

(1) IRMOF-74-I

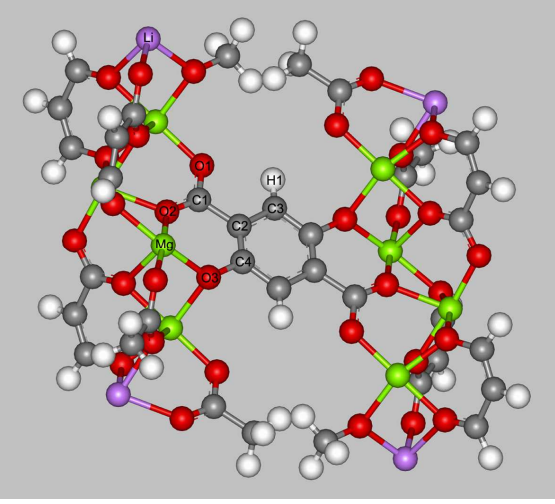

Figure S5. Cluster used for derivation of atomic charges on IRMOF-74-I 
Table S7. Partial atomic charges for IRMOF-74-I

\begin{tabular}{ccccccccc}
\hline Atom name & $\mathrm{Mg}$ & $\mathrm{O} 1$ & $\mathrm{O} 2$ & $\mathrm{O} 3$ & $\mathrm{C} 1$ & $\mathrm{C} 2$ & $\mathrm{C} 3$ & $\mathrm{C} 4$ \\
\hline Charge (e) & 1.3809 & -0.8303 & -0.7337 & -0.8895 & 0.9422 & -0.3507 & -0.1535 & 0.4515 \\
Atom name & $\mathrm{H} 1$ & & & & & & & \\
Charge (e) & 0.1831 & & & & & & & \\
\hline
\end{tabular}

(2) IRMOF-74-V1

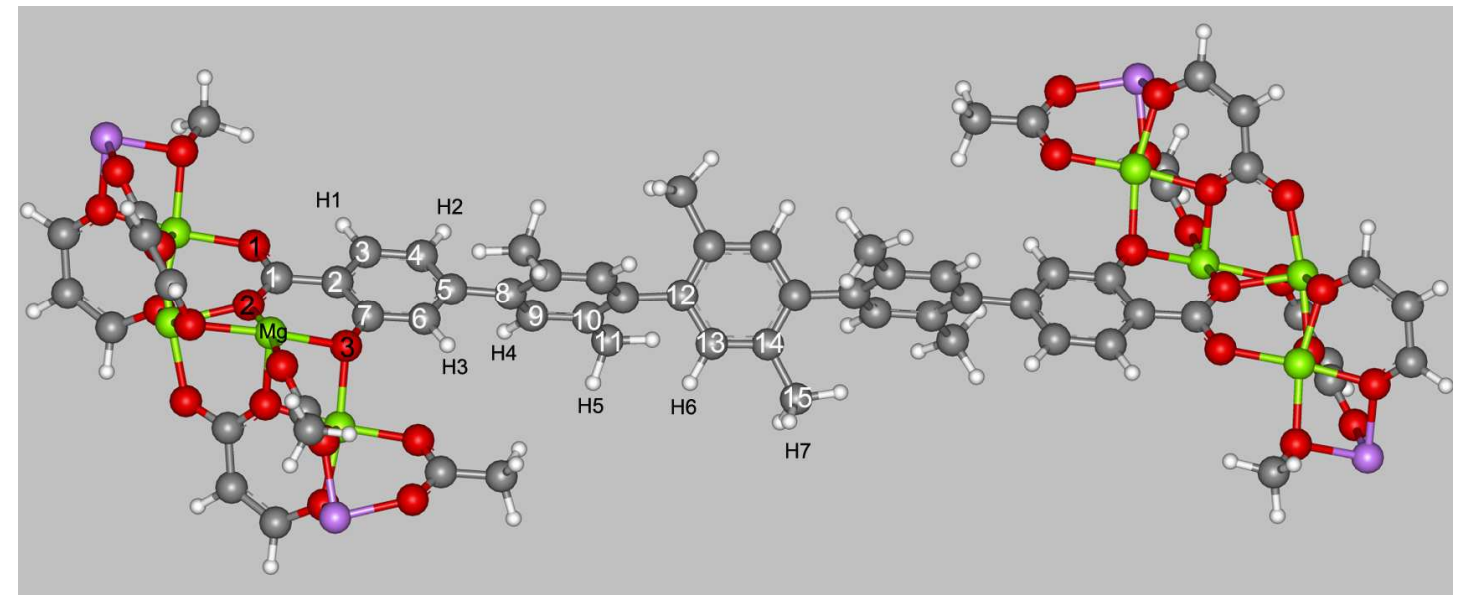

Figure S6. Cluster used for derivation of atomic charges on IRMOF-74-V1

Table S8. Partial atomic charges for IRMOF-74-V1

\begin{tabular}{ccccccccc}
\hline Atom name & $\mathrm{Mg}$ & $\mathrm{O} 1$ & $\mathrm{O} 2$ & $\mathrm{O} 3$ & $\mathrm{C} 1$ & $\mathrm{C} 2$ & $\mathrm{C} 3$ & $\mathrm{C} 4$ \\
\hline Charge (e) & 1.3866 & -0.8015 & -0.7942 & -0.7771 & 0.8923 & -0.3598 & -0.0169 & -0.2723 \\
Atom name & $\mathrm{C} 5$ & $\mathrm{C} 6$ & $\mathrm{C} 7$ & $\mathrm{C} 8$ & $\mathrm{C} 9$ & $\mathrm{C} 10$ & $\mathrm{C} 11$ & $\mathrm{C} 12$ \\
Charge (e) & 0.3558 & -0.3986 & 0.4825 & -0.0404 & -0.2203 & 0.1544 & -0.0842 & 0.0264 \\
Atom name & $\mathrm{C} 13$ & $\mathrm{C} 14$ & $\mathrm{C} 15$ & $\mathrm{H} 1$ & $\mathrm{H} 2$ & $\mathrm{H} 3$ & $\mathrm{H} 4$ & $\mathrm{H} 5$ \\
Charge (e) & -0.2328 & 0.1205 & -0.0608 & 0.1209 & 0.0948 & 0.2025 & 0.0798 & 0.0163 \\
Atom name & $\mathrm{H} 6$ & $\mathrm{H} 7$ & & & & & & \\
Charge (e) & 0.1043 & 0.0170 & & & & & & \\
\hline
\end{tabular}




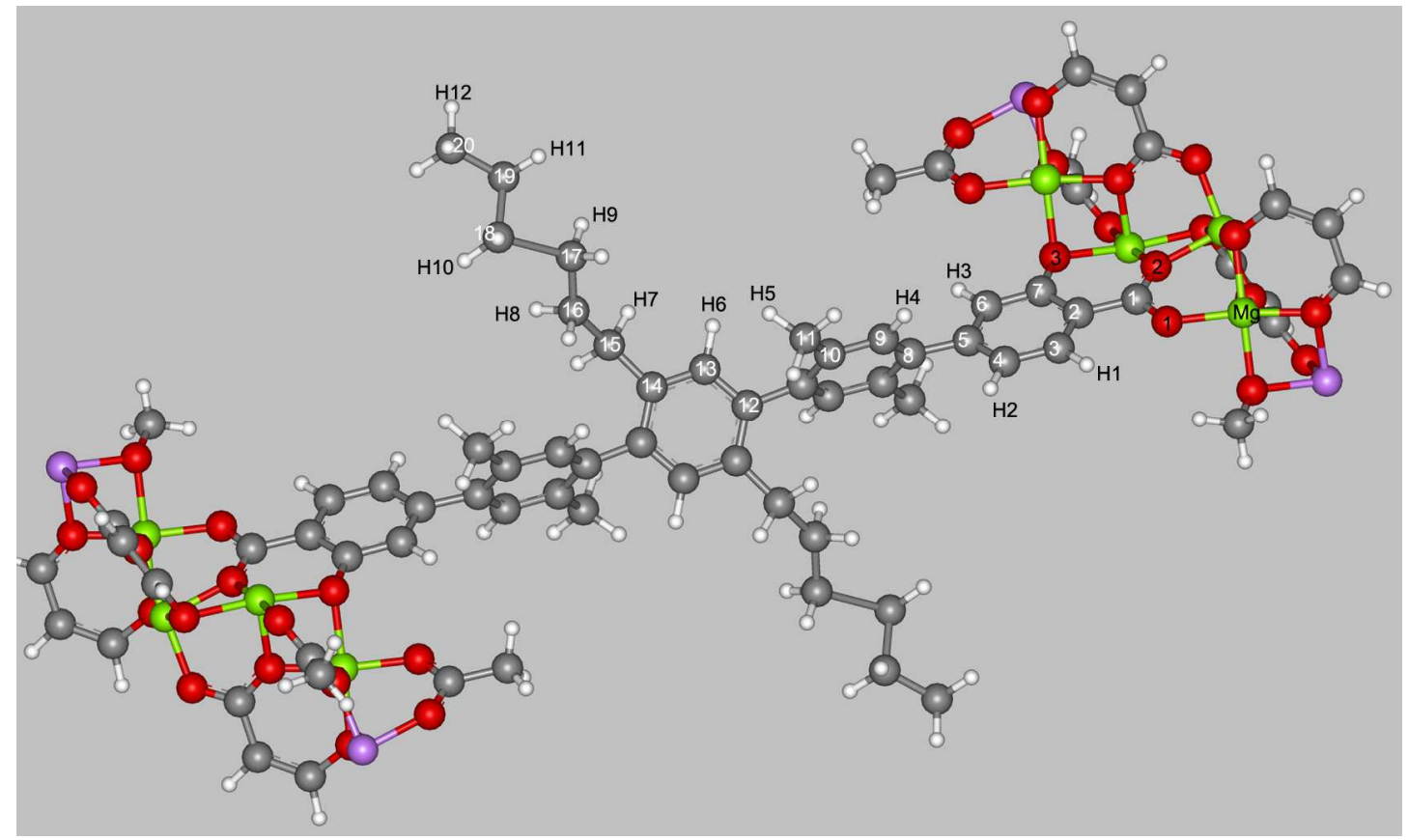

Figure S7. Cluster used for derivation of atomic charges on IRMOF-74-V2

Table S9. Partial atomic charges for IRMOF-74-V2

\begin{tabular}{ccccccccc}
\hline Atom name & $\mathrm{Mg}$ & $\mathrm{O} 1$ & $\mathrm{O} 2$ & $\mathrm{O} 3$ & $\mathrm{C} 1$ & $\mathrm{C} 2$ & $\mathrm{C} 3$ & $\mathrm{C} 4$ \\
\hline Charge (e) & 1.3313 & -0.756 & -0.7526 & -0.7781 & 0.8463 & -0.3517 & -0.0008 & -0.2849 \\
Atom name & $\mathrm{C} 5$ & $\mathrm{C} 6$ & $\mathrm{C} 7$ & $\mathrm{C} 8$ & $\mathrm{C} 9$ & $\mathrm{C} 10$ & $\mathrm{C} 11$ & $\mathrm{C} 12$ \\
Charge (e) & 0.3182 & -0.3522 & 0.4332 & -0.0359 & -0.2290 & 0.113 & -0.0663 & 0.1664 \\
Atom name & $\mathrm{C} 13$ & $\mathrm{C} 14$ & $\mathrm{C} 15$ & $\mathrm{C} 16$ & $\mathrm{C} 17$ & $\mathrm{C} 18$ & $\mathrm{C} 19$ & $\mathrm{C} 20$ \\
Charge (e) & -0.2872 & -0.0399 & 0.1403 & 0.0852 & -0.0912 & -0.296 & 0.3783 & -0.1206 \\
Atom name & $\mathrm{H} 1$ & $\mathrm{H} 2$ & $\mathrm{H} 3$ & $\mathrm{H} 4$ & $\mathrm{H} 5$ & $\mathrm{H} 6$ & $\mathrm{H} 7$ & $\mathrm{H} 8$ \\
Charge (e) & 0.1123 & 0.1160 & 0.2122 & 0.0890 & 0.0203 & 0.1326 & -0.0414 & -0.0295 \\
Atom name & $\mathrm{H} 9$ & $\mathrm{H} 10$ & $\mathrm{H} 11$ & $\mathrm{H} 12$ & & & & \\
Charge (e) & 0.0278 & 0.1003 & -0.0810 & 0.0077 & & & & \\
\hline
\end{tabular}

(4) HA

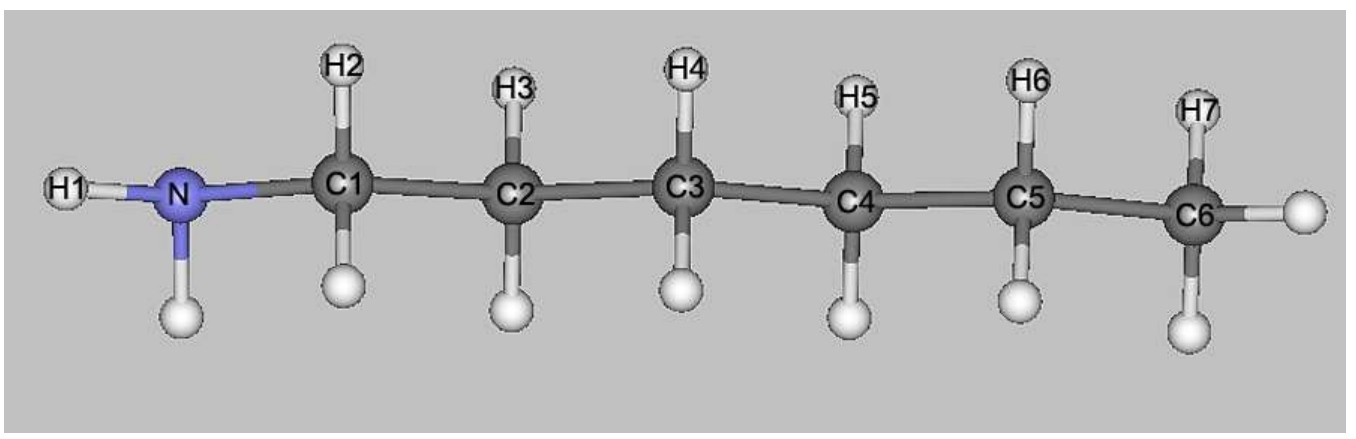

Figure S8 Atom name of hexylamine (HA) 
Table S10. Partial atomic charges for hexylamine (HA)

\begin{tabular}{ccccccccc}
\hline Atom name & $\mathrm{N}$ & $\mathrm{C} 1$ & $\mathrm{C} 2$ & $\mathrm{C} 3$ & $\mathrm{C} 4$ & $\mathrm{C} 5$ & $\mathrm{C} 6$ & $\mathrm{H} 1$ \\
\hline Charge (e) & -1.1380 & 0.6173 & -0.1581 & 0.1010 & 0.1393 & 0.1008 & -0.0776 & 0.3887 \\
Atom name & $\mathrm{H} 2$ & $\mathrm{H} 3$ & $\mathrm{H} 4$ & $\mathrm{H} 5$ & $\mathrm{H} 6$ & $\mathrm{H} 7$ & & \\
Charge (e) & -0.0659 & 0.0037 & -0.0502 & -0.0497 & -0.0345 & 0.0102 & &
\end{tabular}

(5) DGA

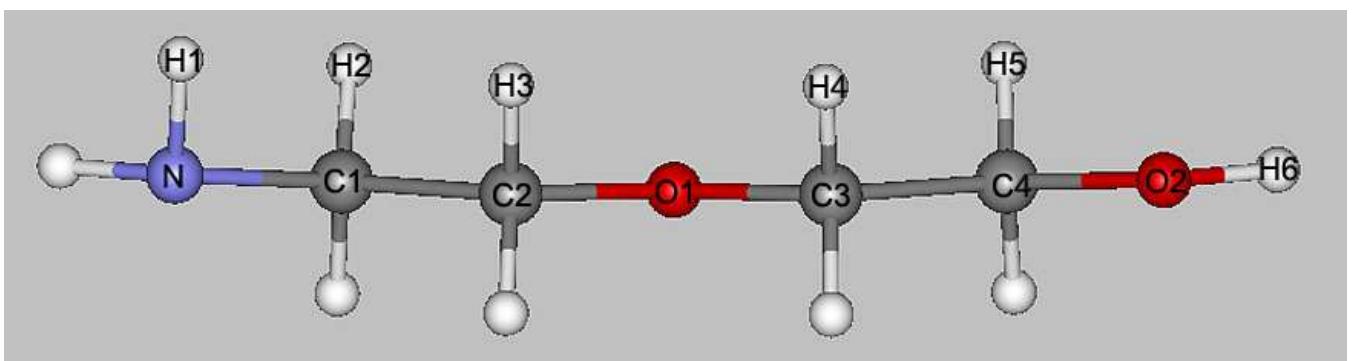

Figure S9. Atom name of diglycolamine (DGA)

Table S11. Partial atomic charges for diglycolamine (DGA)

\begin{tabular}{ccccccccc}
\hline Atom name & $\mathrm{N}$ & $\mathrm{O} 1$ & $\mathrm{O} 2$ & $\mathrm{C} 1$ & $\mathrm{C} 2$ & $\mathrm{C} 3$ & $\mathrm{C} 4$ & $\mathrm{H} 1$ \\
\hline Charge (e) & -1.2183 & -0.7606 & -0.8011 & 0.5536 & 0.4517 & 0.5498 & 0.3547 & 0.4178 \\
Atom name & $\mathrm{H} 2$ & $\mathrm{H} 3$ & $\mathrm{H} 4$ & $\mathrm{H} 5$ & $\mathrm{H} 6$ & & & \\
Charge (e) & -0.0668 & -0.0558 & -0.0572 & -0.0296 & 0.4534 & & &
\end{tabular}

\subsection{Evaluation of $\Delta G$ between AA side chains and IRMOF-74-I}

A thermodynamic cycle in Figure S10 was conducted to calculate the binding free energy $(\Delta G)$ of amino acid (AA) side chains to IRMOF-74-I. In this cycle, $\Delta G_{2} \equiv 0$ and $\Delta G=\Delta G_{3}-\Delta G_{1} . \Delta G$ is defined as the difference between the free energy change of complexation of AA and IRMOF-74-I (i.e. introducing AA into the pore channel of IRMOF-74-I, $\Delta G_{3}$ ) and the free energy of solvating AA in bulk water (i.e. solvation free energy of AA, $\left.\Delta G_{1}\right)$. 

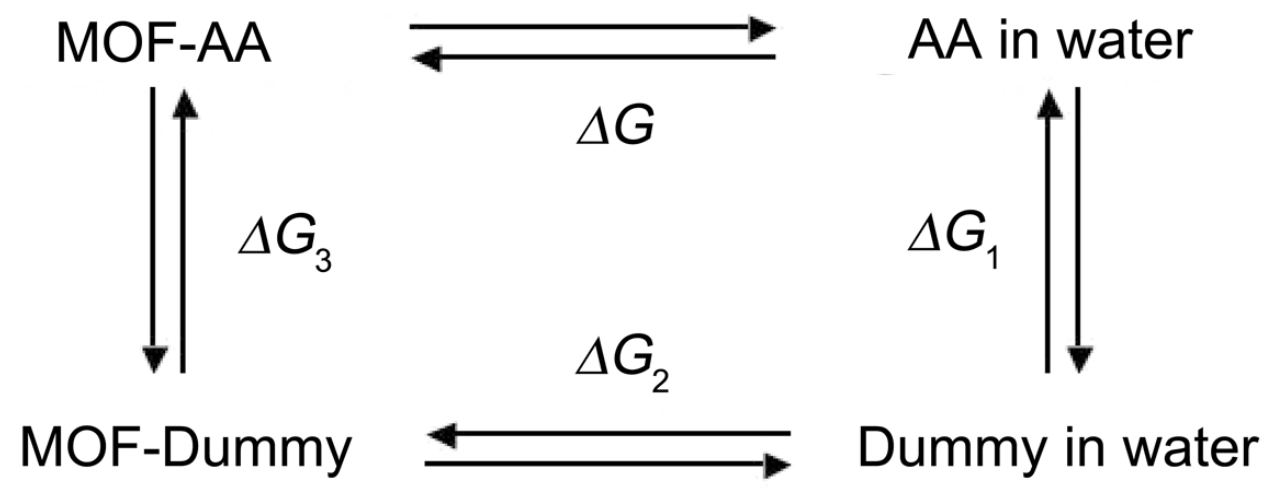

Figure S10. Thermodynamic cycle for free energy calculations of binding amino acid (AA) side chains to IRMOF-74-I.

\section{References}

(1) Kyte, J.; Doolittle, R. F. A Simple Method for Displaying the Hydropathic Character of a Protein. J. Mol. Biol. 1982, 157, 105-132.

(2) Wimley, W. C.; White, S. H. Experimentally Determined Hydrophobicity Scale for Proteins at Membrane Interfaces. Nat. Struct. Mol. Biol. 1996, 3, 842-848.

(3) Hessa, T.; Kim, H.; Bihlmaier, K.; Lundin, C.; Boekel, J.; Andersson, H.; Nilsson, I.; White, S. H.; von Heijne, G. Recognition of Transmembrane Helices by the Endoplasmic Reticulum Translocon. Nature 2005, 433, 377-381.

(4) Shirts, M. R.; Pitera, J. W.; Swope, W. C.; Pande, V. S. Extremely Precise Free Energy Calculations of Amino Acid Side Chain Analogs: Comparison of Common Molecular Mechanics Force Fields for Proteins. J. Chem. Phys. 2003, 119, 5740-5761.

(5) Hornak, V.; Abel, R.; Okur, A.; Strockbine, B.; Roitberg, A.; Simmerling, C. Comparison of Multiple Amber Force Fields and Development of Improved Protein Backbone Parameters. Proteins: Struct., Funct., Genet. 2006, 65, 712-725.

(6) Deng, H.; Grunder, S.; Cordova, K. E.; Valente, C.; Furukawa, H.; Hmadeh, M.; Gándara, F.; Whalley, A. C.; Liu, Z.; Asahina, S. Large-Pore Apertures in a Series of Metal-Organic Frameworks. Science 2012, 336, 1018-1023. 